\title{
Electric Power Network State Tracking from Multirate Measurements
}

\author{
Boris A. Alcaide-Moreno, Member, IEEE, Claudio R. Fuerte-Esquivel, Senior \\ Member, IEEE, Mevludin Glavic, and Thierry Van Cutsem, Fellow, IEEE
}

\begin{abstract}
This paper proposes a novel tracking state estimator to process both fast-rate synchronized phasor and slowrate SCADA measurements. The former are assumed to be in limited number. The latter are exploited as and when they arrive to the control center. In order to restore observability, after each execution of the tracking state estimator, forecasted SCADA measurements are used as pseudo-measurements in the next estimation. An event detection analysis allows assessing if the system is in quasi steady-state. If so, an innovation analysis is performed to identify and eliminate erroneous SCADA measurements. The system state is computed by Hachtel's augmented matrix method. The option of exploiting time-tagged SCADA measurements is also considered. The method is illustrated through detailed dynamic simulations of a test system evolving towards voltage collapse, with and without emergency control.
\end{abstract}

\section{Index Terms}

Tracking state estimation, SCADA, synchrophasor measurements, constrained least-squares, event detection, innovation analysis.

\section{INTRODUCTION}

$\mathbf{S}$ TATE estimation constitutes the backbone of energy management systems. It is an essential tool for real-time monitoring of power system time-varying conditions [1]. Those dynamic changes in the state, resulting from the continuous variation of loads and renewable generation, or the occurrence of disturbances, need to be monitored continuously for early detection of potential operational problems and taking the corresponding preventive or corrective remedial actions. This motivates the execution of power system state estimation at shorter intervals of time, using all the available measurements, for better situational awareness.

Early proposals of Tracking State Estimation (TSE), for better following of the network state evolution, can be traced back to [2], [3], [4], and [5] based on the technology available at that time. Those publications suggested the use of a Kalman filter or extended the techniques developed for static estimation to the time-varying case. Slow dynamic changes in the system state can be tracked through Kalman filter-based state forecasting approaches that require a complete set of Supervisory Control And Data Acquisition (SCADA) measurements in order to carry out successive estimations, as proposed in [6] and [7]. The detection and identification of bad data or sudden changes in the operation conditions is proposed in [8] based on an innovation analysis which relies on comparing real measurements with their corresponding computed values. This type of comparison is also adopted in [4] and [6] for detecting bad data through logical check routines instead of using a test for skewness of the distributions generated by an innovation analysis [8].

B.A. Alcaide-Moreno and C.R. Fuerte-Esquivel are with the Electrical Engineering Faculty, Universidad Michoacana de San Nicolás de Hidalgo (UMSNH), Morelia, Michoacán, México (e-mail: balcaide@dep.fie.umich.mx, cfuerte@umich.mx). This work was supported by CONACyT, México, under grant 321846.

M. Glavic is with the Department of Electrical Engineering and Computer Science, University of Liège, B-4000 Liège, Belgium (e-mail: mevludin.glavic@ulg.ac.be)

T. Van Cutsem is with the Fund for Scientific Research (FNRS) at the same department (e-mail: t.vancutsem@ulg.ac.be). 
Traditionally, measurements used for power system monitoring are collected by low updating rate SCADA systems via Remote Terminal Units (RTUs) [1]. Estimations based on those measurements could not be accurate enough in capturing how the system states evolve in time. On the other hand, Phasor Measurement Units (PMUs), which provide synchronized measurements at 30 or more samples per second, are expected to improve the efficiency and accuracy in which the time-varying nature of the power system is captured [9]. Depending on the number of PMUs installed in the network, linear state estimation [10], a fully dynamic state estimator [11] or a complete measurement of all system state variables could be the foundation to enable a true real-time monitoring [12].

For economic and technical reasons the penetration of PMUs is low and far from being rich enough for ensuring full system observability [9], [12]. Therefore, as long as there are not enough PMUs deployed in power networks, it will be relevant to take advantage of both SCADA and scarce PMU measurements to track the system evolution. In many systems PMUs have been deployed with the aim of monitoring electromechanical oscillations. SCADA measurements are typically not used to this purpose. This paper demonstrates the possibility of combining both types of measurements for further applications and improved situational awareness. Clearly, the accuracy and the rate at which the changing system can be tracked depends on the number and accuracy of the available real-time measurements, and particularly the PMUs. Potential applications may vary from one system to another, but typical examples involve, non exhaustively, the tracking of transmission line overloads, the initialization "on the fly" of time-domain simulations, or the wide-area detection of voltage instability [13], [14].

Several approaches combining SCADA and PMU measurements have been proposed to estimate the network state. Leaving existing state estimation software unchanged, Ref. [15] proposed a post-processing linear state estimator using synchrophasor measurements to enhance accuracy. Following the idea to keep existing state estimation software unchanged, it was proposed in [16] to process separately SCADA (by existing software) and phasor measurements (as an additional estimation module), with techniques from fusion theory to combine both results. In [14], bus injection estimates obtained from previous state reconstructions were used as pseudo-measurements to restore observability. The idea of combining SCADA and synchrophasor measurements has been further elaborated in a number of recent publications. A weighted least absolute value based tracking estimator has been proposed in [17] that uses the incoming PMU measurements and the last set of estimated SCADA measurements as pseudo-measurements. In [18], a linear state estimation has been proposed assuming a rich deployment of PMUs but not enough to make the system observable; observability is then restored by computing missing measurements using network equations. In Ref. [19], missing SCADA measurements are extrapolated through the exponential moving average method between SCADA successive communications, and an optimization problem is solved using an extended Kalman filter. In [20], as in [21], the states of PMU unobservable buses are interpolated taking advantage of regularly updated SCADA measurements [21] or when a disturbance is detected considering recursive a priori state information [20]. Most of these approaches, however, consider that SCADA and PMU measurements are taken at one single point in time. They neglect the important issue of time skew affecting the various real-time measurements.

The drawback mentioned above is overcome in [22], [23], within the context of tracking the network state, by simultaneously processing SCADA and PMU measurements as and when they arrive to the control center: the last received SCADA measurements, the synchrophasor measurements and pseudomeasurements stemming from the previous TSE run are processed at each TSE execution instead of assuming that all SCADA (resp. PMU) measurements are collected in a single snapshot in time intervals of $\Delta_{\text {scada }}$ (resp. $\Delta_{P M U}$ ) seconds. Following this line of reasoning, this paper extends the work presented in [22], [23] with the following specific contributions:

1) Pseudomeasurements are treated in a more uniform manner and their values are obtained by a prediction based on previous TSE results. This is achieved by using a known prediction method in the new context where SCADA measurements are used as and when they arrive. A heuristic procedure, giving the minimum mean variance percentage errors, to determine parameters of SCADA measurement predictor is introduced. 
2) We demonstrate that time-tagging SCADA measurements are beneficial for (state estimation in general and) tracking state estimation in particular and consider both time-tagged and not time-tagged SCADA measurements in the state estimation process. A suitable way of processing time-tagged measurements is proposed considering the way in which they arrive to the control center.

3) An approach for detecting the occurrence of a significant change in system operation is proposed based on incremental changes between successive PMU samples. The proposed procedure relies on the assumption that synchrophasor measurements are exempt from large errors, other than those resulting from system dynamics. This assumption is realistic provided that PMU measurements have been preprocessed for data conditioning and bad data repairing, as proposed in [24].

4) Event detection and innovation analysis are performed to discriminate between a change in the system state and the occurrence of erroneous SCADA measurements. The proposed test is performed using active and reactive power flows to avoid false detection of sudden changes in the system operating point.

The paper is structured as follows. The principles behind the proposed TSE are explained in Section II, while the mathematical formulation and its solution method are detailed in Section III. Illustrative examples of the TSE performance and application are reported in Section IV. The main features of the approach and directions for further research are summarized in Section V.

\section{PRINCIPLES OF THE TSE METHOD}

\section{A. Time delays affecting SCADA measurements}

Today's standard practice in industry for static state estimation assumes that all types of measurements are taken at one point in time [9]. However, the various SCADA measurement values are collected at different times and transmitted to the control center with different delays. Owing to this lack of synchronization, when the system is not in steady state, the solution provided by a static state estimator significantly differs from the true, changing operating state of the monitored system. This is known as the time skew problem.

The SCADA measurement gathering process is shown in Fig. 1, which illustrates the communication latency associated with the measurements from two substations [22]. In a given substation, the various measurement devices (transducers) are "visited" cyclically with a time period of $\Delta_{i}$, which has an order of magnitude of one second, and the measured values are stored sequentially in the memory of the RTU. At a given time $t^{*}$, the RTU of the $i$-th substation sends the contents of its memory to the SCADA system of the control center, which is received with a transmission time delay $\tau_{i}$ that is generally below half a second. Note that the transmitted $j$-th measured value is the one stored at the previous time $t^{*}-\delta_{j i}$ where $\delta_{j i}$ is a value in the interval $\left[0, \Delta_{i}\right]$. While measurements are gathered continuously within the substation, the RTU only communicates with the SCADA system of the control center at regular time intervals $T_{i}$ that may lie in between two and five seconds, depending on the technology, the age of the equipment, etc.

\section{B. Principles of the proposed method}

Unlike a static state estimator, the TSE processes measurements at short intervals of time in order to follow the changing state.

A distinct procedure is applied to SCADA measurements which are time tagged, and to those which are not. Namely, in existing SCADA systems some measurements are time-tagged (for example, the measurements taken by digital fault recorders) [25]. In this context, time-tagging all SCADA measurements would be beneficial in general and for tracking state estimation in particular. Furthermore, this could be achieved at low cost. What would be needed is to upgrade existing RTUs with GPS receivers (the cost of the receivers, as of the beginning of 2017, is approximately US \$20). Through checking major RTU providers it is observed that newer RTUs are coming with GPS receiver embedded (although still 


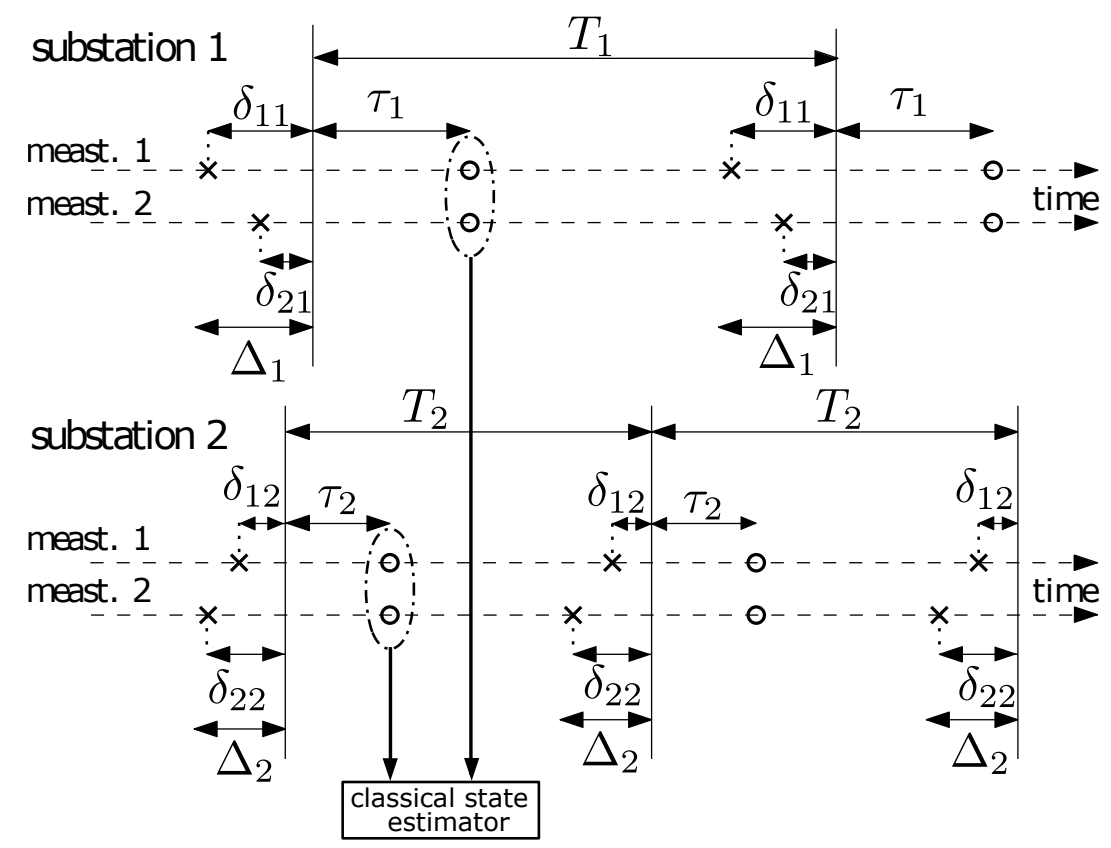

Figure 1. Delays on SCADA measurements. $\times$ indicates the time at which a measurement is taken, $\circ$ the time at which it is received by the control center.

with primary function to time-tag fault recorders). The way time-tagged measurements are transmitted to SCADA masters is to be considered. However, we believe the communication protocols used in existing SCADA systems would easily allow this extension without the need to upgrade existing communications infrastructure, which would make the solution costly. The need for time-tagging SCADA measurements was mentioned by WECC (Western Electricity Coordination Council, covering Western parts of USA and Canada) plans in [26].

Consider first the case of (classical) non time-tagged SCADA measurements. They are processed as and when they are received, as sketched in Fig. 2. At a given time $t$, the set of processed measurements is composed of the most recent synchronized (bus voltage and branch current) phasor measurements as well as the new SCADA (voltage magnitude, active or reactive power) measurements that have been received since the last TSE execution, i.e. in the time interval $\left[t-T_{r}, t\right]$. Note that SCADA measurements used at that time are affected by delays $\delta_{j i}+\tau_{i}+\epsilon_{i}$ where $\epsilon_{i}$ is the dead time between the arrival of a measurement and its processing. In order to minimize the effect of time skew, especially when the system is undergoing some dynamic changes, all SCADA measurements received before time $t-T_{r}$ are no longer used.

If time-tagged SCADA measurements are available, the time skew problem can be mitigated. Indeed, when a measurement arrives with the indication that it was taken at time $t^{\prime}<t$, it can be assigned to the time interval $\left[t-(p+1) T_{r}, t-p T_{r}\right]$ such that $-(p+1) T_{r} \leq t^{\prime}-t<-p T_{r}$. Then, the measurements assigned to that interval are processed with a delay in order to ascertain that no further measurement will be assigned to that interval. This is illustrated in Fig. 3. where it is shown that the TSE performed at time $t$ processes the SCADA measurements collected in the interval $\left[t-4 T_{r}, t-3 T_{r}\right]$ and the PMUs relative to time $t-3 T_{r}$. Thus, $p=3$ in this example. With $T_{r}$ in the order of one second, this delay appears reasonable, considering that TSE is used for monitoring, not immediate control purposes. On the other hand, higher accuracy is to be expected since the SCADA measurements used are less dispersed over time.

Even though all PMU measurements are exploited, the use of only a fraction of the whole set of SCADA measurements in each TSE generally results in unobservability. Pseudo-measurements must be added to restore observability. The latter, referred to as predicted SCADA measurements, are obtained from the most recent TSEs by predicting the values of the SCADA measurements through a time series analysis. 


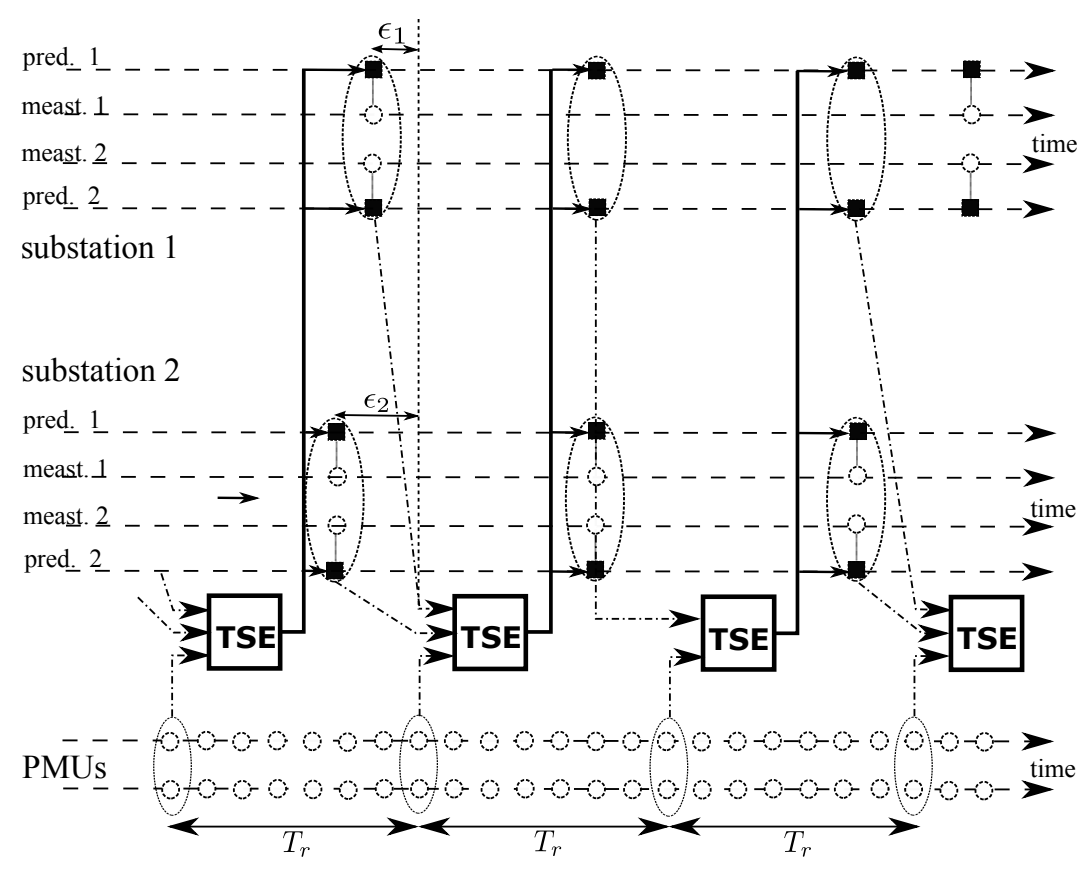

Figure 2. Use of SCADA, PMU and predicted SCADA measurements in the proposed TSE. $\circ$ indicates a SCADA measurement, $\mathbf{a}$ predicted value.

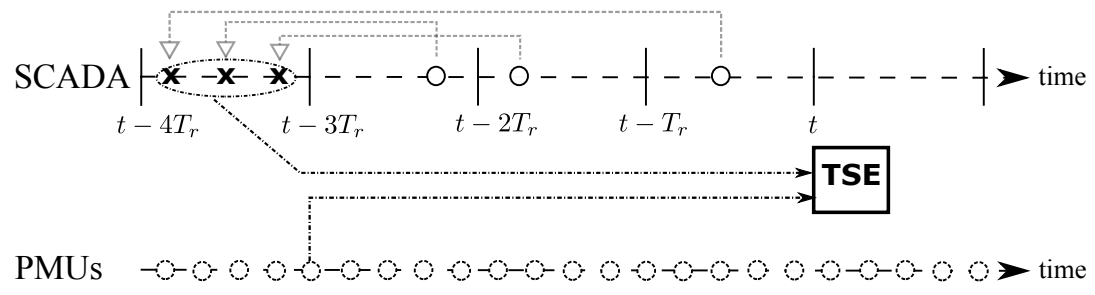

Figure 3. Processing of time-tagged SCADA measurements. $\circ$ indicates the time at which a measurement is received, $\times$ the time at which it was taken.

Based on the above mentioned information, the TSE processes at time $t$ the subset of SCADA measurements collected in the time interval $\left[t-T_{r}, t\right]$ (resp. $\left[t-(p+1) T_{r}, t-p T_{r}\right]$ if time-tagged SCADA mesurements are exploited), the synchrophasor measurements relative to time $t$ (resp. $t-p T_{r}$ ), and the predicted SCADA measurements, to obtain a new estimate of the state vector at time $t$ (resp. $t-p T_{r}$ ). These new values of state variables are then used to calculate the estimated values of all quantities measured by the SCADA system, which in turn are employed to predict the values of all SCADA measurements at time $t+T_{r}$ (resp. $t-(p-1) T_{r}$ ).

Lastly, zero bus injections (known with infinite accuracy) are included in the formulation as constraints to improve the accuracy of the proposed method.

\section{TSE MATHEMATICAL FORMULATION}

\section{A. Measurement model}

Let $m$ be the total number of SCADA measurements. The measurement model is made up of the following equations:

$$
\begin{aligned}
\boldsymbol{z}_{s}(k) & =\boldsymbol{D}_{k} \boldsymbol{h}_{s}(\boldsymbol{x}(k))+\boldsymbol{e}_{s} \\
\boldsymbol{z}_{p}(k) & =\boldsymbol{h}_{p}(\boldsymbol{x}(k))+\boldsymbol{e}_{p} \\
\overline{\boldsymbol{z}}_{s}(k / k-1) & =\boldsymbol{\phi}\left[\boldsymbol{h}_{s}(\hat{\boldsymbol{x}}(k / k)), \overline{\boldsymbol{z}}_{s}(k / k-1)\right]+\overline{\boldsymbol{e}}_{s}
\end{aligned}
$$




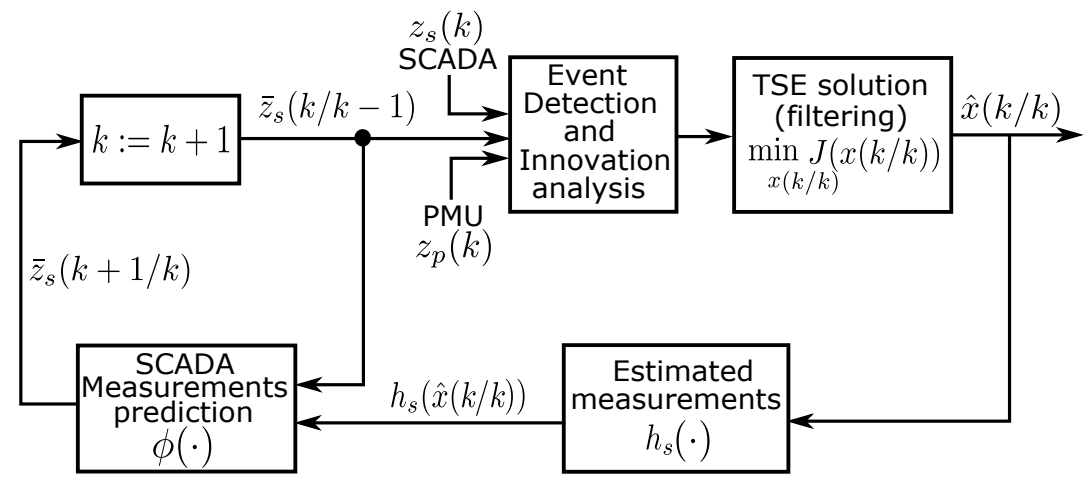

Figure 4. Main stages of proposed tracking state estimation.

where $\boldsymbol{x}(k)$ is the state vector to be estimated at time $k, \boldsymbol{z}_{s}(k)$ (resp. $\boldsymbol{z}_{p}(k)$ ) is the vector of SCADA (resp. PMU) measurements available at time $k, \boldsymbol{h}_{s}(\cdot)\left(\operatorname{resp} . \boldsymbol{h}_{p}(\cdot)\right)$ are functions relating SCADA (resp. PMU) measurements with the state vector, $\boldsymbol{D}_{k}$ is built from an $m \times m$ unit matrix by removing the rows corresponding to the SCADA measurements not present at time $k, \overline{\boldsymbol{z}}_{s}(k / k-1)$ is the vector of predicted SCADA measurements available at time $k$, which uses previous TSEs, $\phi(\cdot)$ is a prediction function, and $\boldsymbol{e}_{s}, \boldsymbol{e}_{p}$ and $\overline{\boldsymbol{e}}_{s}$ are unknown vectors of measurement and prediction errors.

The proposed TSE procedure consists of three steps, shown in Fig. 4. SCADA measurement prediction, event detection and innovation analysis, and estimation of the system state.

\section{B. SCADA measurement prediction}

In this stage the values of all SCADA measurements are predicted for time $k+1$. Various methods can be used to that purpose. The choice is not very critical (in [22] even a simple "persistence model" was considered) in so far as prediction is over a time interval of a few seconds, and TSE is not aimed at tracking faster transients, not relevant to power system monitoring from a control center.

Among the various time series analysis methods, Holt's Linear (HL) approach has been selected for its simplicity [6], [27]. It relies on the estimates at the current time $k$ and at the previous time $k-1$ according to:

$$
\overline{\boldsymbol{z}}_{s}(k+1 / k)=\boldsymbol{F}_{k} \boldsymbol{h}_{s}(\hat{\boldsymbol{x}}(k / k))+\boldsymbol{g}_{k}
$$

where:

$$
\begin{aligned}
\boldsymbol{F}_{k} & =\alpha(1+\beta) \boldsymbol{I} \\
\boldsymbol{g}_{k} & =(1+\beta)(1-\alpha) \overline{\boldsymbol{z}}_{s}(k / k-1)-\beta \boldsymbol{a}_{k-1}+(1-\beta) \boldsymbol{b}_{k-1} \\
\boldsymbol{a}_{k} & =\alpha\left(\boldsymbol{h}_{s}(\hat{\boldsymbol{x}}(k / k))\right)+(1-\alpha) \overline{\boldsymbol{z}}_{s}(k / k-1) \\
\boldsymbol{b}_{k} & =\beta\left(\boldsymbol{a}_{k}-\boldsymbol{a}_{k-1}\right)+(1-\beta) \boldsymbol{b}_{k-1} .
\end{aligned}
$$

$\boldsymbol{F}_{k}$ is an estimate of the series "level" and $\boldsymbol{g}_{k}$ of its slope, both at time $k$, while $\overline{\boldsymbol{z}}_{s}(k / k-1)$ is the previous vector of predicted SCADA measurements. On the other hand, $\alpha$ is a smoothing parameter while $\beta$ is the smoothing parameter for the trend.

\section{Innovation analysis and event detection}

In static state estimation, anomalous data are most often detected and identified through the (normalized) residuals, which involve the difference between the measured and the estimated values of a SCADA measurement. Dynamic, as well as tracking, state estimation offers an additional data validation, through the innovation vector, i.e. the difference between the predicted and the measured values of a measurement. 
This test, referred to as innovation analysis, can be performed before the state vector is estimated; it is thus possible to avoid the estimated state to be "contaminated" by the bad data. This is important in so far as the contamination effect of a bad measurement persists in the subsequent state estimations (through the pseudo-measurements $\bar{z}_{s}$ ).

On the other hand, it may happen that the components of the innovation vector are abnormally large due to a significant change in the system operating state, which is not reflected by the prediction (4). Tracking such changes is precisely the objective of TSE. In this case, larger components of the innovation (and residual) vectors must not be attributed to bad measurements; they have to be accepted as the result of changes taking place in the system, whose effects will hopefully die out in the subsequent TSE runs.

There is thus a need to detect the occurrence of a change in system operation. In this paper a simple method for event detection is considered. To detect changes in operating state, incremental changes between successive PMU samples can be checked against a proper threshold. However, keeping in mind the way phasors are calculated [9], more precisely the fact that phase angles can change as the data window advances in time owing to the phasor rotation, the test is performed on equivalent active and reactive power flow measurements, as well as on voltage magnitude measurements. Denoting by $V_{i}<\theta_{i}$ the voltage phasor at bus $i$ and $I_{i j} \angle \delta_{i j}$ the current phasor in a line connected to that bus, the corresponding active and reactive power flows are:

$$
\begin{aligned}
& P_{i j}^{e q}=V_{i} I_{i j} \cos \left(\theta_{i}-\delta_{i j}\right) \\
& Q_{i j}^{e q}=V_{i} I_{i j} \sin \left(\theta_{i}-\delta_{i j}\right) .
\end{aligned}
$$

The variance of the equivalent $P_{i j}^{e q}$ measurement is given by:

$$
\sigma_{P_{i j}}^{2}=\left[\frac{\partial P_{i j}}{\partial V_{i}}\right]^{2} \sigma_{V_{i}}^{2}+\left[\frac{\partial P_{i j}}{\partial \theta_{i}}\right]^{2} \sigma_{\theta_{i}}^{2}+\left[\frac{\partial P_{i j}}{\partial I_{i j}}\right]^{2} \sigma_{I_{i j}}^{2}+\left[\frac{\partial P_{i j}}{\partial \delta_{i j}}\right]^{2} \sigma_{\delta_{i j}}^{2}
$$

where $\sigma_{V_{i}}$ is the standard deviation of the noise affecting $V_{i}, \sigma_{\theta_{i}}$ the one of $\theta_{i}, \sigma_{I_{i j}}$ the one of $I_{i j}$ and $\sigma_{\delta_{i j}}$ the one of $\delta_{i j}$. A similar formula applies to $Q_{i j}^{e q}$.

Accordingly, the event detection test at time $k$ is:

$$
v_{P_{i j}}=\left|\frac{P_{i j}^{e q}(k)-P_{i j}^{e q}(k-1)}{\sigma_{P_{i j}}}\right| \stackrel{?}{>} \gamma
$$

where $k-1$ and $k$ denote two successive PMU sampling times, and $\gamma$ is a properly chosen threshold. A similar test applies to $Q_{i j}^{e q}$, while changes in voltage magnitude can be merely checked on the measurements directly:

$$
v_{V_{i}}=\left|\frac{V_{i}(k)-V_{i}(k-1)}{\sigma_{V_{i}}}\right| \stackrel{?}{>} \gamma
$$

After a change in operating state has been detected, the states obtained by TSE at a few subsequent times (typically a few seconds) are marked as being insufficiently accurate, due to inaccuracy in the forecasting method during the transient response and time skew in SCADA measurements.

On the other hand, if no significant change has been detected, the incoming SCADA measurements can be checked for gross errors using the above mentioned innovation vector. The test applied to the $i$-th measurement is:

$$
v_{s i}(k)=\left|\frac{\left[\boldsymbol{z}_{s}(k)\right]_{i}-\left[\boldsymbol{D}_{k} \overline{\boldsymbol{z}}_{s}(k / k-1)\right]_{i}}{\sigma_{s i}}\right| \stackrel{?}{<} \psi
$$

where $\sigma_{s i}$ is the standard deviation of the noise affecting that measurement, and $\psi$ is a properly chosen threshold. The measurements for which the test fails are removed from $\boldsymbol{z}_{s}$. There is no resulting unobservability issue owing to the presence of the pseudo-measurements $\overline{\boldsymbol{z}}_{s}$. 


\section{TSE solution (filtering step) method}

The correction step yields the estimate of the state vector $\hat{\boldsymbol{x}}(k / k)$ at time $k$ by processing the measurements involved in Eqs. (1 - 3). By assuming Gaussian error distributions, the maximum likelihood estimate of $\boldsymbol{x}(k / k)$ is found by minimizing the objective function:

$$
J=\frac{1}{2} \boldsymbol{r}_{s}^{T} \boldsymbol{D}_{k} \boldsymbol{R}^{-1} \boldsymbol{D}_{k}^{T} \boldsymbol{r}_{s}+\frac{1}{2} \boldsymbol{r}_{p}^{T} \boldsymbol{U}^{-1} \boldsymbol{r}_{p}+\frac{1}{2} \overline{\boldsymbol{r}}_{s}^{T} \boldsymbol{M}^{-1} \overline{\boldsymbol{r}}_{s}
$$

subject to equality constraints corresponding to zero power injections in transit buses:

$$
\boldsymbol{f}(\boldsymbol{x}(k / k))=\mathbf{0}
$$

and the residuals of respectively $\boldsymbol{z}_{s}, \boldsymbol{z}_{p}$ and $\overline{\boldsymbol{z}}_{s}$ :

$$
\begin{array}{r}
\boldsymbol{r}_{s}-\boldsymbol{z}_{s}(k)+\boldsymbol{D}_{k} \boldsymbol{h}_{s}(\boldsymbol{x}(k / k))=\mathbf{0} \\
\boldsymbol{r}_{p}-\boldsymbol{z}_{p}(k)+\boldsymbol{h}_{p}(\boldsymbol{x}(k / k))=\mathbf{0} \\
\overline{\boldsymbol{r}}_{s}-\overline{\boldsymbol{z}}_{s}(k / k-1)+\boldsymbol{h}_{s}(\boldsymbol{x}(k / k))=\mathbf{0} .
\end{array}
$$

In the objective (15), $R, U$ and $M$ are the covariance matrices associated with the various noises involved in Eqs. (1- 3).

This optimization problem is solved using Hachtel's augmented matrix method [28], which is known for its good numerical conditioning [1]. The latter requires solving a sequence of sparse, symmetric, linear systems $(i=1,2, \ldots)$ :

$$
\left[\begin{array}{lllll}
\boldsymbol{D}_{k} \boldsymbol{R} \boldsymbol{D}_{k}^{T} & \mathbf{0} & \mathbf{0} & \boldsymbol{D}_{k} \boldsymbol{H}_{s} & \mathbf{0} \\
\mathbf{0} & \boldsymbol{U} & \mathbf{0} & \boldsymbol{H}_{p} & \mathbf{0} \\
\mathbf{0} & \mathbf{0} & \boldsymbol{M} & \boldsymbol{H}_{s} & \mathbf{0} \\
\boldsymbol{H}_{s}^{T} \boldsymbol{D}_{k}^{T} & \boldsymbol{H}_{p}^{T} & \boldsymbol{H}_{s}^{T} & \mathbf{0} & \boldsymbol{F}^{T} \\
\mathbf{0} & \mathbf{0} & \mathbf{0} & \boldsymbol{F} & \mathbf{0}
\end{array}\right]\left[\begin{array}{l}
\boldsymbol{\mu}_{s}^{i} \\
\boldsymbol{\mu}_{p}^{i} \\
\boldsymbol{\mu}_{f}^{i} \\
\Delta \boldsymbol{x}^{i} \\
\boldsymbol{\lambda}^{i}
\end{array}\right]=\left[\begin{array}{l}
\boldsymbol{z}_{s}(k)-\boldsymbol{D}_{k} \boldsymbol{h}_{s}\left(\boldsymbol{x}^{i-1}(k / k)\right) \\
\boldsymbol{z}_{p}(k)-\boldsymbol{h}_{p}\left(\boldsymbol{x}^{i-1}(k / k)\right) \\
\overline{\boldsymbol{z}}_{s}(k / k-1)-\boldsymbol{h}_{s}\left(\boldsymbol{x}^{i-1}(k / k)\right) \\
\mathbf{0} \\
-\boldsymbol{f}\left(\boldsymbol{x}(k / k)^{i-1}\right)
\end{array}\right]
$$

and incrementing the state vector according to:

$$
\boldsymbol{x}^{i}(k / k)=\boldsymbol{x}^{i-1}(k / k)+\Delta \boldsymbol{x}^{i}
$$

until a convergence criterion is satisfied. The iterations are initialized by setting $\boldsymbol{x}^{0}(k / k)$ to the solution $\hat{\boldsymbol{x}}(k-1 / k-1)$ of the TSE at the previous time step, or from a flat voltage profile if the TSE iterations are re-initialized.

A rectangular components formulation has been used for the network equations, with the state vector $\boldsymbol{x}$ being composed of real and imaginary parts of the complex bus voltages.

\section{Simulation Results}

\section{A. Test system}

The so-called Nordic test system shown in Fig. 5] and documented in [29] was chosen to assess the performance of the proposed TSE in significantly disturbed operating conditions.

Its model includes 74 buses, 20 generators, 102 branches, among which 20 step-up and 22 distribution transformers. The study focuses on tracking the system evolution after the outage of line 4032-4044, due to a three-phase solid fault at bus 4032, cleared after five cycles. Two scenarios are presented in this paper. In the first one, the system experiences long-term voltage instability [29] under the effect of transformer Load Tap Changers (LTCs) and generator Over-Excitation Limiters (OELs). In the second one, stability is restored with an undervoltage load shedding of $100 \mathrm{MW}$ at bus 1041 and $200 \mathrm{MW}$ at bus 1044, performed in several steps [29].

The set of measurements considered in both scenarios is composed of two multi-channel PMUs and 139 SCADA measurements, located in the system as shown in Fig. 5. The PMU at bus 4044 provides 


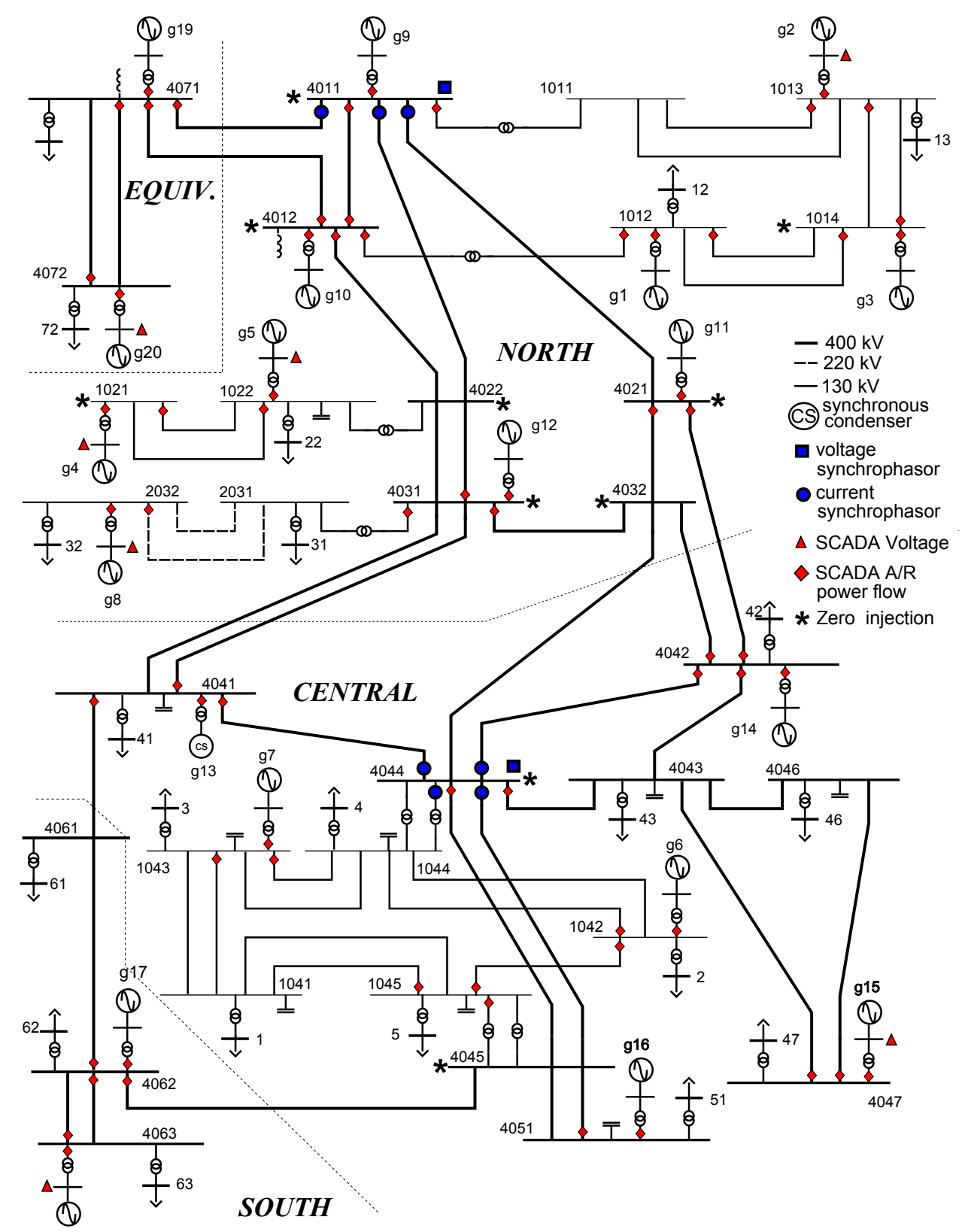

Figure 5. Nordic system with SCADA and PMU measurement configuration.

one voltage and four current synchrophasors, while the one at bus 4011 provides one voltage and three current synchrophasors. On the other hand, SCADA measurements involve seven voltage magnitudes at generator buses and 66 pairs of active/reactive power flows.

The "exact" values of all these measurements were obtained from dynamic simulations of the system response (one for each scenario), considering detailed models of the generators and their controllers, including OELs and LTCs [29].

Next, these values were processed to add time delays in accordance with the gathering of SCADA measurements shown in Fig. 1. Namely, it was considered that the $i$-th RTU transmits its set of measurements to the control center every $T_{i}$ seconds, with $2 \leq T_{i} \leq 5$. A random communication delay of $\tau_{i}$ seconds was added, with $0.1 \leq \tau_{i} \leq 0.5$. This delay corresponds to the total delay including: fixed delay of transducer, data processing, multiplexing, link propagation delay, random delay jitter, and data rate of the link [30]. This delay range was chosen to cover a mix of communication links present in todays power system infrastructure (ranging from telephone lines to fiber-optic cables [30]). It was also considered 
that measurements in the $i$-th substation are collected at different time instants, leading to a delay of $\delta_{j i}$ seconds for the $j$-th measurement, with $0.1 \leq \delta_{j i} \leq 0.9$.

Finally, Gaussian distributed measurement noises were added to obtain the final "measured" values. The standard deviations of SCADA voltage magnitude and power flow measurements were set to $\sigma_{s, V}=$ $0.00167 \mathrm{pu}$ and $\sigma_{s, F}=0.0025 S_{n o m}^{p u}$ pu, respectively, where $S_{n o m}^{p u}$ is the nominal power of the branch in per unit. For PMUs, the standard deviations of both real and imaginary components were set to $\sigma_{p, V}=0.2 \sigma_{s, V}$ for voltages and $\sigma_{p, I}=0.2 \sigma_{s, F}$ for currents.

The TSE also processes ten pairs of zero injections at buses 4011, 4012, 1014, 1021, 4022, 4021, 4031, 4032, 4044 and 4045 through the constraints (16).

The 22 distribution buses and the corresponding transformers were not included in the model handled by the TSE, although they were present in the dynamic simulation, experiencing numerous tap changes. This led to a TSE involving 32 transmission and 20 generator buses, and 80 branches. The measurement redundancy is $(139+20) / 103=1.54$ with SCADA data only, and 1.72 with the synchrophasor measurements. These values are deemed moderate, if not low [1].

Network topology was assumed to be updated in the TSE execution that follows the line tripping. On the other hand, the network state was tracked without knowing about the OEL activations, the LTC tap changes and the load curtailments.

\section{B. Accuracy Indices}

In order to assess the accuracy at time $k$ and over the whole simulation, the following indices have been considered.

The Mean Absolute Percentage Errors (MAPE) evaluate the voltage magnitude mean absolute error (in percent):

$$
\begin{gathered}
M A P E(k)=\frac{1}{N} \sum_{i=1}^{N} \frac{\left|V_{i}^{e x}-V_{i}^{e s t}\right|}{V_{i}^{e x}} 100 \% \\
M A P E=\frac{1}{T} \sum_{k=1}^{T} \frac{1}{N} \sum_{i=1}^{N} \frac{\left|V_{i k}^{e x}-V_{i k}^{e s t}\right|}{V_{i k}^{e x}} 100 \%
\end{gathered}
$$

where $N=52$ is the number of buses, $T$ is the total number of successive TSE executions, the superscript $e x$ denotes exact values, and ${ }^{e s t}$ estimates.

The Mean Absolute Errors (MAE) assess the accuracy of voltage phase angles (in degrees).

$$
\begin{gathered}
M A E(k)=\frac{1}{N} \sum_{i=1}^{N}\left|\theta_{i}^{e x}-\theta_{i}^{e s t}\right| \\
M A E=\frac{1}{T} \sum_{k=1}^{T} \frac{1}{N} \sum_{i=1}^{N}\left|\theta_{i k}^{e x}-\theta_{i k}^{e s t}\right|
\end{gathered}
$$

\section{Predicted SCADA measurements}

For the sake of simplicity and computational efficiency, the $M$ covariance matrix relative to predicted SCADA measurements was approximated by a diagonal matrix whose entries were chosen heuristically as follows.

The standard deviation associated with each predicted measurement was assumed to be $K$ times the corresponding SCADA standard deviation $\left(\sigma_{s, V}\right.$ or $\left.\sigma_{s, F}\right)$. $K$ was chosen by provisionally taking as prediction of a SCADA measurement the value estimated at the previous TSE execution, as in [22]. $K$ was varied over a wide range, and the value leading to the smallest MAPE index was identified as $K=3.5$, which has been used in the subsequent simulations. 


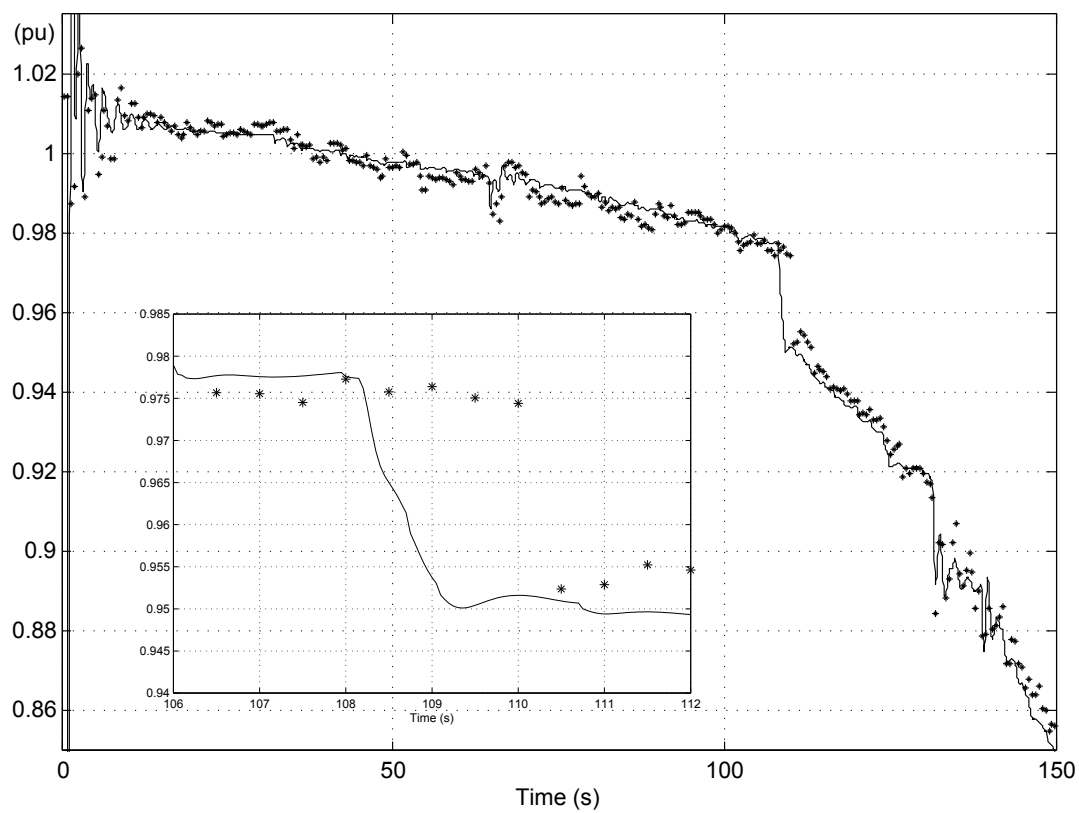

Figure 6. Exact and tracked voltage magnitude at bus 1042 using classical SCADA and synchrophasor measurements; voltage collapse scenario.

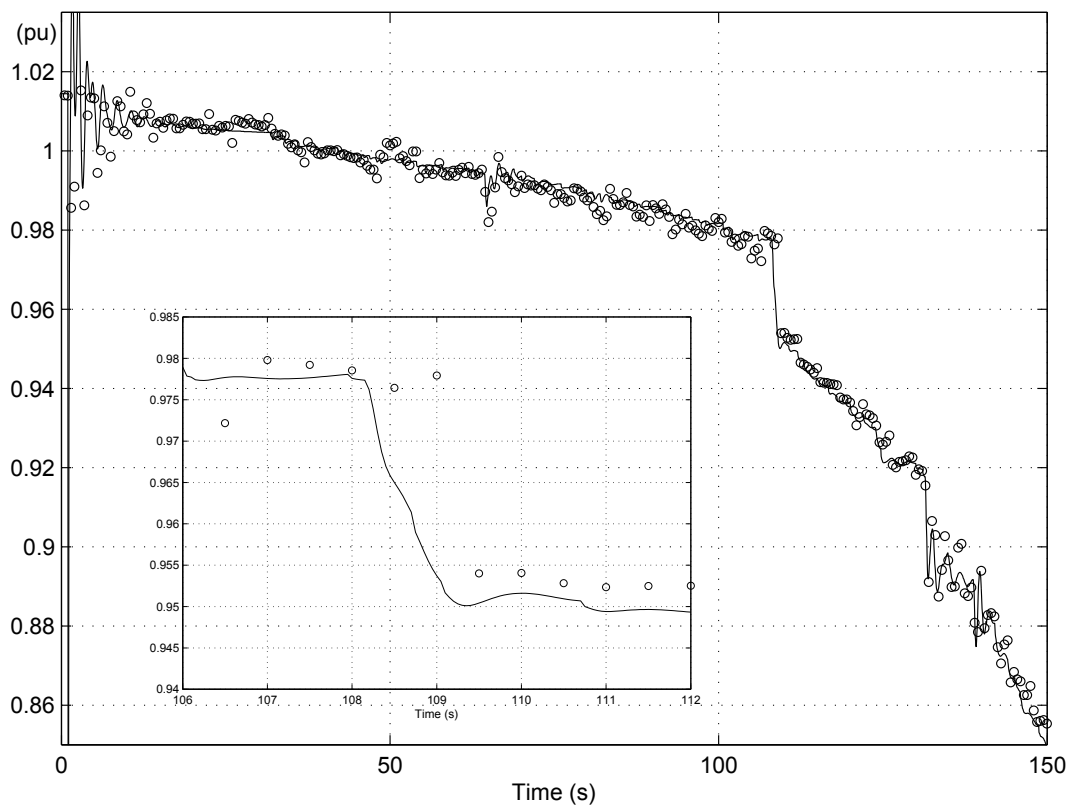

Figure 7. Exact and tracked voltage magnitude at bus 1042 using time-tagged SCADA and synchrophasor measurements; voltage collapse scenario.

Using this value of $K$, the $\alpha$ and $\beta$ parameters of the HL prediction (5.8) were also varied and the combination leading again to the smallest MAPE value was identified as $\alpha=0.6$ and $\beta=0.5$, also used in the subsequent simulations.

The results obtained with these settings are already very satisfactory. The fact remains that higher accuracy could be achieved with a more rigorous covariance analysis.

\section{Accuracy of TSE}

In all tests reported here, the period of TSE execution $T_{r}$ was set to 0.5 second.

The exact and tracked long-term evolutions of the most affected voltage magnitude in the voltage collapse scenario are shown in Fig. 6 for the case where classical SCADA measurements are processed, 


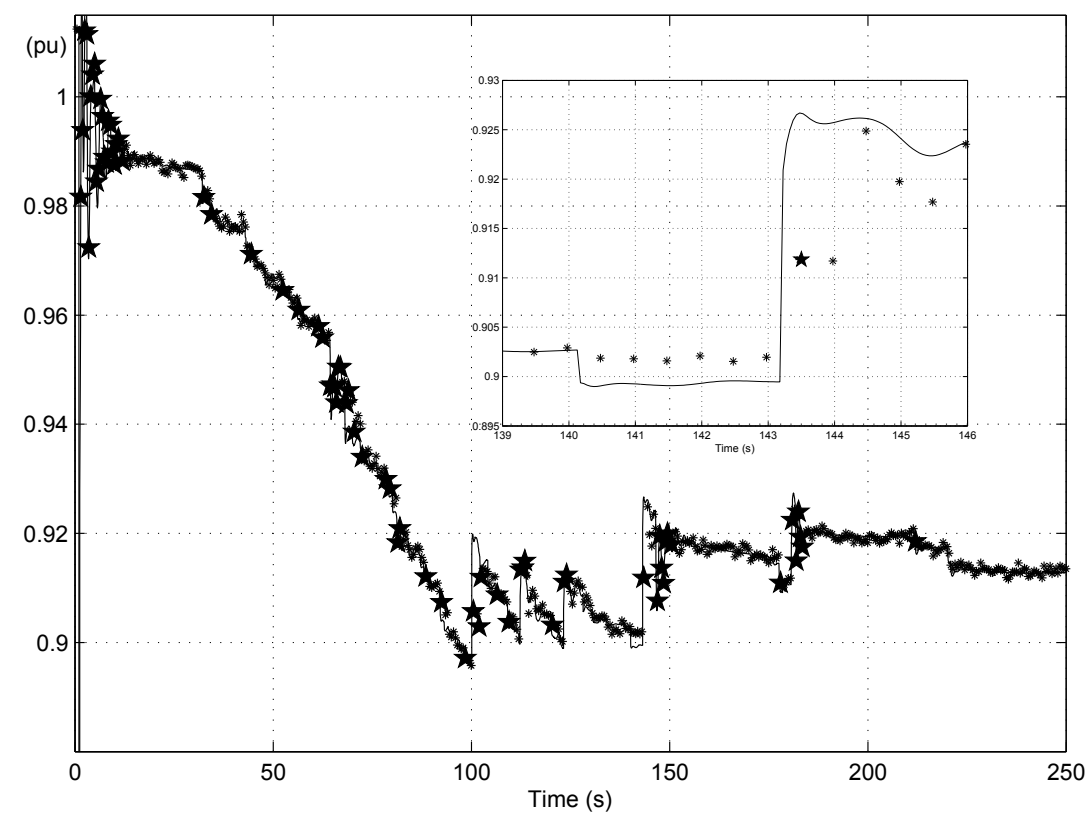

Figure 8. Exact and tracked voltage magnitude at bus 1041 using classical SCADA and synchrophasor measurements; load shedding scenario.

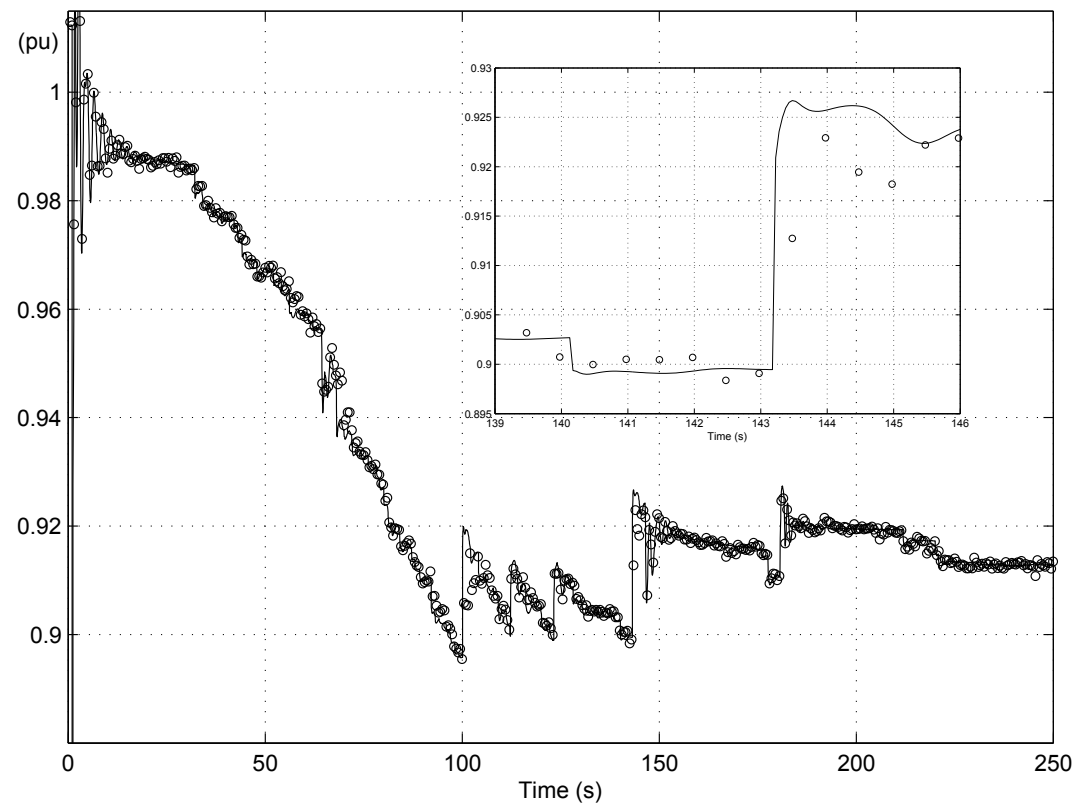

Figure 9. Exact and tracked voltage magnitude at bus 1041 using time-tagged SCADA and synchrophasor measurements; load shedding scenario.

and in Fig. 7 when their time-tagged counterparts are used. The final voltage collapse (corresponding to a loss of synchronism of g6) is not shown, to preserve legibility. In both figures, the exact evolution is shown with solid line, while the estimated values are shown with asterisks (resp. circles) when the SCADA measurements do not come (resp. come) with time stamps. The corresponding results in the case with stabilization by load shedding are given in Figs. 8 and 9 , respectively.

The plots provide evidence that the proposed TSE is able to track the network state evolution, in particular when changes take place in the system, for instance due to remedial actions. Expectedly, the state estimation is not satisfactory during the large transients that follow the fault itself, but after some 10 to 15 seconds, TSE realigns on the system evolution.

The zoomed views in Figs. 6 to 9 confirm that after a sudden change in the system (in the shown case, an OEL activation), the tracked evolution follows the exact one with a delay, caused by the non-synchronized 
Table I

MAPE AND MAE INDICES

\begin{tabular}{rcc}
\hline & $M A P E(\%)$ & $M A E(\mathrm{deg})$ \\
\hline No disturbance & 0.057854 & 0.036848 \\
Voltage Collapse scenario & 0.121980 & 0.063582 \\
Same with time-tagged SCADA & 0.107020 & 0.056033 \\
Load Shedding scenario & 0.082208 & 0.044838 \\
Same with time-tagged SCADA & 0.076298 & 0.043544 \\
\hline
\end{tabular}

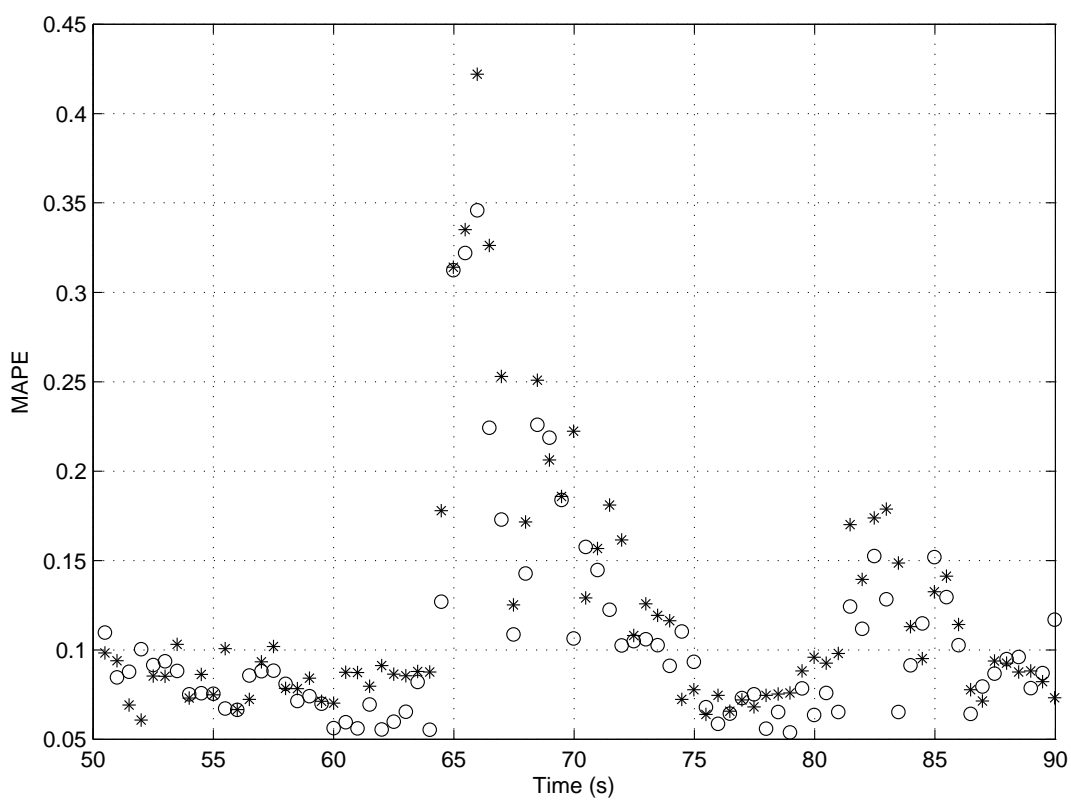

Figure 10. $M A P E(k)$ index considering classical SCADA (asterisks) and time-tagged SCADA (circles) measurements; load shedding scenario.

SCADA measurements and the absence of PMUs near the bus of concern. This delay, however, is rather short when classical SCADA measurements are used, and slightly reduced when time-tagged SCADA measurements are processed as described in Section II.

The values of MAPE and MAE indices are given in Table $\mathrm{I}$ in the aforementioned two scenarios as well as when the system is in steady state. The latter case is for reference, since the TSE errors result from measurement noise only (no transient, no time skew). For the above mentioned reason, the first ten seconds after fault clearing were not included in the calculation of the indices. These results confirm that using time-tagged SCADA measurements improves the overall accuracy of TSE.

Moreover, a comparison of the $M A P E(k)$ indices with and without time-tagged SCADA measurements is shown in Fig. 10 for the load shedding scenario. The higher index values occur at times in which sudden changes take place. This justifies to label inaccurate the estimates provided by a number of TSE executions after an event has been detected.

Lastly, in order to assess how the accuracy of measurements affects the proposed TSE, the scenario related to the stability restoration through undervoltage load shedding has been simulated considering larger values of standard deviations for both SCADA and PMU measurements. Standard deviation values of $\sigma_{s, V}=0.004 \mathrm{pu}$ and $\sigma_{s, F}=0.008 \mathrm{pu}$ have been considered for SCADA measurements as proposed in [1]. On the other hand, the standard deviations of the errors for both voltage and current phasor measurements are set at $\sigma_{p, V}=0.002 \mathrm{pu}$ for voltages and $\sigma_{p, I}=(1 / 5)(0.0025) \sigma_{s, F}$ for currents. The tracking state of the voltage magnitude at node 1041 by using classical SCADA and synchrophasor measurements is depicted in Fig. 11, with values of MAPE and MAE indices given by 0.097775 and 0.071034 , respectively. A comparison of these indices with respect to those obtained by considering lower values of standard deviations (values of 0.082208 and 0.044838 for the MAPE and MAE indices, respectively) indicates that 


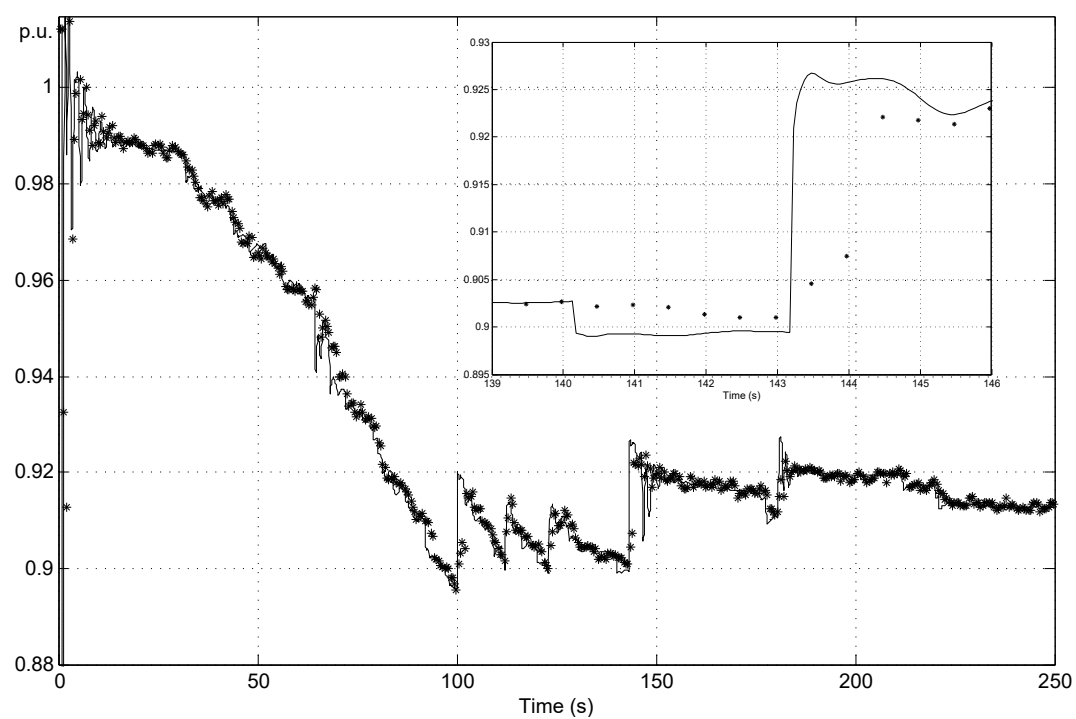

Figure 11. Exact and tracked voltage magnitude at bus 1041 by using classical SCADA and synchrophasor measurements with larger standard deviations; load shedding scenario.

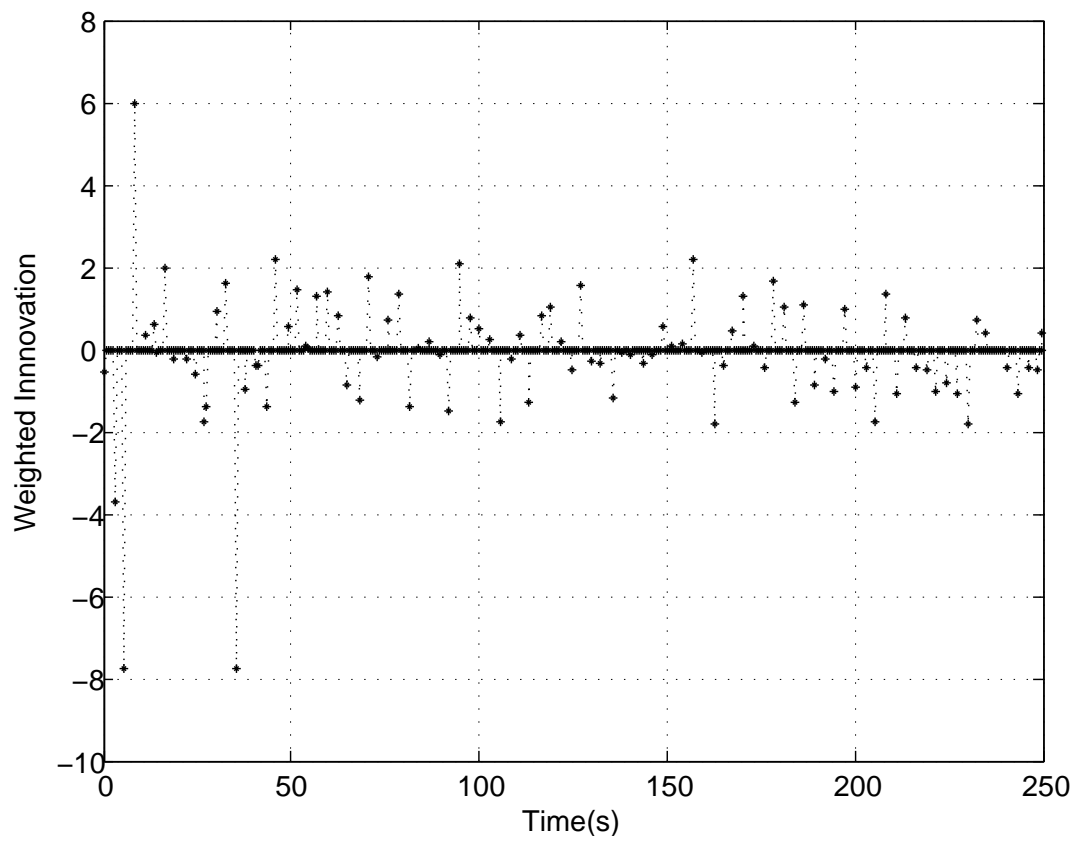

Figure 12. Innovation values for active power flow $P_{4041-4031}$ measurement.

the performance of the proposed approach is still quite good even when using larger standard deviations.

\section{E. Bad data and event detection}

The innovation analysis and event detection is illustrated by considering the case of a gross error of $10 \sigma_{s, F}$ affecting the active power flow $P_{4041-4031}$ at $t=35.1 \mathrm{~s}$, in the load shedding scenario. The successive innovation values for this measurement, involved in the test (14), are shown in Fig. 12, from which a gross error is easily observed at $t=35.5 \mathrm{~s}$. At the same time instant, the tests (12) and (13) do not reveal any significant change in the operating state: the largest $v_{P_{i j}}$ value for all PMUs is 0.9604 . Hence, the large innovation value is attributed to a bad measurement.

On the contrary the large innovation values observed at $t=3.0,5.5$, and $8.5 \mathrm{~s}$, respectively, are due to the transients triggered by the fault. At these times, the tests (12) and (13) are positive, indicating that the system undergoes significant changes instead of a gross measurement error (and, hence, the estimates are flagged as inaccurate during a few TSE executions after each of these times). 
Further research is needed in order to define appropriate values of $\gamma$ in $(12,13)$ and $\psi$ in (14). In the tests reported here, the values $\psi=4.5$ and $\gamma=6.5$ were found to provide a good compromise between false alarms and non detections.

The way in which the values of $\gamma$ and $\psi$ affect the correct identification of events and of bad data can be approached from the following theoretical sensitivity analysis viewpoint. A high value of $\gamma$ means that only events producing large changes in the innovation vector will be identified such that the alarm to perform a control action for some events occurring in the system will not be sent to the operator. This will undermine the situational awareness of the grid operator. On the other hand, a low value of $\gamma$ results in false alarms because small variations in the innovation vector, which are related to normal quasi-steady state changes, can be erroneously associated with the occurrence of an event.

A similar line of reasoning applies to the values of $\psi$ in the context of the detection of gross errors in measurements. Hence, a high value of $\psi$ means that some gross errors to be undetected, such that the tracking state estimator will process these gross errors as good SCADA measurements. In order to avoid an erroneous TSE by the incorrect preprocessing of raw data, an additional bad data analysis is performed after each TSE execution. The bad data detection and identification are addressed with the chisquared and largest normalized residual tests. Note that a re-estimation is required after each identification which could lead to computer times incompatible with the on-line requirements. On the other hand, good measurements will be considered as gross errors if a small value of $\psi$ is adopted in the simulation such that the removed measurements from $z_{s}$ will reduce the dimension of (17).

\section{F. TSE assessment through Monte-Carlo simulations}

A more systematic assessment of the TSE accuracy was performed through Monte-Carlo simulations. A set of simulations was built, differing by the random noise applied to the measurements, as well as by random transmission delays $\tau_{i}$ in the interval $[0.1,0.5] \mathrm{s}$. For each voltage magnitude and power flow provided with a SCADA measurement, the mean and standard deviations of the corresponding $s$ estimates was computed, for each of the $T$ TSE executions, as follows:

$$
\begin{aligned}
\mu_{j}(k) & =\frac{1}{s} \sum_{i=1}^{s} e_{j, i}(k) \\
\sigma_{j}(k) & =\sqrt{\frac{1}{s} \sum_{i=1}^{s}\left(e_{j, i}(k)-\mu_{j}(k)\right)^{2}}
\end{aligned}
$$

where:

$$
e_{j, i}(k)=h_{j}\left(\hat{\boldsymbol{x}}_{i}(k / k)\right)-h_{j}(\boldsymbol{x}(k))
$$

is the estimation error of the $j$-th measured quantity, at the $k$-th discrete time $(k=1, \ldots, T)$, provided by the $i$-th simulation $(i=1, \ldots, s)$. Then, for each measured quantity, a global mean and standard deviation were computed over all $T$ discrete times according to:

$$
\begin{aligned}
\bar{\mu}_{j} & =\frac{1}{T} \sum_{k=1}^{T} \mu_{j}(k) \\
\bar{\sigma}_{j} & =\sqrt{\frac{1}{T} \sum_{k=1}^{T} \sigma_{j}(k)^{2} .}
\end{aligned}
$$

The mean (29) and variance (30) were computed after each simulation run and the Monte-Carlo simulations were stopped when the observed changes in these values were deemed negligible over last 50 consecutive simulation runs. In the case of concern here, it was found that 500 simulations were enough.

This analysis was performed in the two aforementioned scenarios as well as in the case with no disturbance. 
Table II

STANDARD DEVIATIONS OF MEASUREMENT NOISE AND ESTIMATION ERRORS FOR THREE MEASURED ACTIVE POWER FLOWS (IN PU)

\begin{tabular}{lcccccc}
\hline \multirow{2}{*}{ Line } & Meas. & \multicolumn{2}{c}{ No } & \multicolumn{2}{c}{ Load Shedding } & \multicolumn{2}{c}{ Voltage } \\
& noise & disturb. & \multicolumn{2}{c}{ scenario } & \multicolumn{2}{c}{ Collapse } \\
& $\sigma_{s, F j}$ & $\bar{\sigma}_{j}$ & $\bar{\mu}_{j}$ & $\bar{\sigma}_{j}$ & $\bar{\mu}_{j}$ & $\bar{\sigma}_{j}$ \\
\hline \hline $4047-4043$ & 0.0350 & 0.0317 & 0.0025 & 0.0289 & -0.0031 & 0.0322 \\
$2032-2031$ & 0.0125 & 0.0095 & 0.0021 & 0.0120 & 0.0041 & 0.0109 \\
$1043-1041$ & 0.0087 & 0.0077 & 0.0018 & 0.0068 & 0.0017 & 0.0077 \\
\hline
\end{tabular}

Table III

NUMBER OF ITERATIONS OF HACHTEL'S METHOD

\begin{tabular}{c|cccc}
\hline & \multicolumn{4}{|c}{ Number of time steps with } \\
Scenario & 2 iter. & 3 iter. & 4 iter. & 5 iter. or more \\
\hline Voltage collapse & 280 & 46 & 2 & 0 \\
Load shedding & 465 & 33 & 2 & 0 \\
\hline
\end{tabular}

For a sample of three active power flows, Table [II shows the standard deviation of the measurement noise $\sigma_{s, F j}$ and the $\bar{\mu}_{j}$ and $\bar{\sigma}_{j}$ statistics. It can be seen that the bias $\bar{\mu}_{j}$ is very small. The values of $\bar{\sigma}_{j}$ being smaller than those of $\sigma_{s, F j}$ reveal the filtering capability of the proposed TSE in spite of the significant system transients and the small number of PMUs.

In the presented results, the outage of the line 4032-4044 caused a series of system changes in terms of several generator OEL activations (in the voltage unstable case) and load shedding in the stabilized case, which were correctly detected by the proposed approach and the selected value of $\gamma$. It is true that the value of $\gamma$ is system dependent and as a rule of thumb should be determined using a set of credible scenarios with the aim to achieve a good compromise between false alarms and non detections.

\section{G. Convergence of Hachtel's method}

Table III gives an account of the number of iterations of Hachtel's method outlined in Section III-D. The iterations are stopped when $\max _{j}\left|\left[\Delta \boldsymbol{x}^{i}\right]_{j}\right|<10^{-4}$. It can be seen that most of the time, two or three iterations are sufficient. The highest values correspond to states estimated during large transients. This, and the availability of efficient solvers for symmetric sparse matrices allows running TSE very frequently.

\section{H. Comparison with previously proposed approaches}

The suitability of the proposed approach is assessed by reporting the accuracy indices associated with the results obtained with the method of this paper and those obtained by other methods. Hence, the TSE has been performed for the voltage collapse scenario considering the following cases: i) the proposed approach considering time-tagged SCADA measurements and values of $\alpha=0.6$ and $\beta=0.5$; ii) the proposed approach considering time-tagged SCADA measurements and the values of $\alpha=0.8$ and $\beta=0.5$ suggested in [6]; and iii) the approach suggested in [22]. Note that this approach does not consider timetagged SCADA measurements nor SCADA measurements prediction, and only the last set of SCADA measurements are used to perform the TSE. The MAPE and MAE indices for each study case are displayed in Table IV. These results clearly show that the more accurate TSE is obtained by the proposed approach with the values of $\alpha$ and $\beta$ determined according to the procedure described in Section IV-C.

\section{CONCLUSION}

A novel state estimation has been presented, aimed at tracking the changes of network state. The main feature is the simultaneous processing of measurements gathered at different rates: SCADA measurements as and when they arrive, and synchrophasor measurements available at much higher rate. In this work the 
Table IV

ACCURACY INDICES FOR STUDY CASES

\begin{tabular}{rcc}
\hline & $M A P E(\%)$ & $M A E(\mathrm{deg})$ \\
\hline Proposed approach & 0.107020 & 0.056033 \\
Reference [6] & 0.11933 & 0.071659 \\
Reference [22] & 0.14722 & 0.067607 \\
\hline
\end{tabular}

emphasis was on taking advantage of a relatively small number of synchronized measurements, a situation that is likely to prevail for quite some time in many power systems.

Predicted SCADA measurements are used to ensure observability. A combined analysis of synchronized measurement variations and innovation vectors has been proposed to distinguish between sudden changes in system state and gross errors affecting the SCADA measurements.

Furthermore, time-tagged SCADA measurements can be exploited. This mitigates the time skew effect but requires running the TSE with a delay.

In the tests, heuristic weights have been assigned to the predicted SCADA measurements. It is certainly worth investigating how a more accurate covariance matrix could be used instead, although Monte-Carlo simulations confirm noise filtering capabilities even when the aforementioned sub-optimal weights are used.

The reported innovation analysis approach relies on the assumption that there is no bad data affecting synchrophasor measurements, so that their change with time can be used to detect sudden changes in system operating point. It is of interest to deal with bad data on synchrophasor measurements which would have escaped the pre-conditioning [24]. This processing is likely to become easier when a larger proportion of such measurements will be available.

Further extensions of this work will include investigation of how scarce synchrophasor measurements could be used to detect changes in network topology (this functionality is implicitly assumed in proposed approach) following the ideas presented in [31] and [32].

The accuracy of synchrophasor measurements is impaired during strong transient conditions for several reasons, e.g. (i) their accuracy deteriorate when the fundamental frequency differs from the nominal 50 or $60 \mathrm{~Hz}$, (ii) the sampled signal suffers distortion from a pure sine wave, and (iii) errors are introduced by the instrumentation channel components (i.e. instrument transformers, control cables, burdens, filters, and A/D converters). Some of the errors (instrument transformers, control cables, burdens) present even in (quasi) steady-state operating conditions can be efficiently handled by calibration (most PMUs are equipped with the option for external calibration [33]) while some others are difficult to compensate (filters and A/D converters). Reference [33] provides some guidelines on how to handle these errors. Research efforts are devoted by PMU developers to deal with this specific problem as well as with a better characterization of the accuracy during transient conditions, for various applications, not just the tracking state estimation considered in this paper.

Finally, the multirate feature could be extended to even less frequently collected measurements such as data from smart meters in distribution grids.

Nevertheless, the results already show the ability of the proposed method to track the network state evolution even under severe conditions.

\section{REFERENCES}

[1] A. Abur and A. G. Exposito, Power system state estimation: theory and implementation. New York: Marcel Dekker, 2004.

[2] A. Debs and R. E. Larson, "A dynamic state estimator for tracking the state of power sytems," IEEE Trans. Power App. and Syst., vol. PAS-89, pp. 1670-1678, Sep. 1970.

[3] R. Masiello and F. C. Schweppe, "A tracking state estimator," IEEE Trans. Power App. and Syst., vol. PAS-90, pp. 1025-1033, Mar. 1971.

[4] D. M. Falcao, P. A. Cooke, and A. Brameller, "Power system tracking state estimation and bad data processing," IEEE Trans. Power App. Syst., vol. PAS-101, pp. 325-333, Feb. 1982. 
[5] W. W. Kotiuga, "Development of a least absolute value power system tracking state estimator," IEEE Trans. Power App. Syst., vol. PAS-104, pp. 1160-1166, May 1985.

[6] A. M. L. da Silva, M. B. D. C. Filho, and J. F. de Queiroz, "State forecasting in electric power systems," Proc. IEE on Gener, Transm. and Distrib., vol. 30, pp. 237-244, Sep. 1983.

[7] M. Brown, D. C. Filho, and J. C. S. de Souza, "Forecasting-aided state estimation - part i: Panorama," IEEE Trans. Power Syst., vol. 24, pp. 1667-1677, Nov. 2009.

[8] K. Nishiya, J. Hasegawa, and T. Koike, "Dynamic state estimation including anomaly detection and identification for power systems," Proc. IEE on Gener, Transm. and Distrib., vol. 129, pp. 102-108, Sep. 1982.

[9] A. Phadke and J. S. Thorp, Synchronized Phasor Measurements and Their Application. New York: Springer, 2008.

[10] S. Sarri, L. Zanni, M. Popovic, J. Y. L. Boudec, and M. Paolone, "Performance assessment of linear state estimators using synchrophasor measurements," IEEE Trans. Instrum. Meas., vol. 65, no. 3, pp. 535-548, Mar. 2016.

[11] F. Aminifar, M. Shahidehpour, M. Fotuhi-Fituzabad, and S. Kamalinia, "Power system dynamic state estimation with synchronized phasor measurements," IEEE Trans. Instrum. Meas., vol. 63, no. 2, pp. 352-363, Jan. 2014.

[12] P. Zhang, F. Li, and N. Bhatt, "Next-generation monitoring, analysis, and control for the future smart control center," IEEE Trans. Smart Grid, vol. 1, no. 2, pp. 186-192, Sep. 2010.

[13] M. Glavic and T. V. Cutsem, "Wide-area detection of voltage instability from synchronized phasor measurements. part i: Principle," IEEE Trans. Power Syst., vol. 24, no. 3, pp. 1408-1416, Aug. 2009.

[14] M. Glavic and T. Van Cutsem, "Reconstructing and tracking network state from a limited number of synchrophasor measurements," IEEE Trans. Power Syst., vol. 28, pp. 1921-1929, May 2013.

[15] M. Zhou, V. A. Centeno, J. S. Thorp, and G. Phadke, "An alternative for including phasor measurements in state estimators," IEEE Trans. Power Syst., vol. 21, no. 4, pp. 1930-1937, Nov. 2006.

[16] A. S. Costa, A. Albuquerque, and D. Bez, "An estimation fusion method for including phasor measurements into power system real-time modeling," IEEE Trans. Power Syst., vol. 28, no. 2, pp. 1910-1920, May 2013.

[17] M. Gol and A. Abur, "A hybrid state estimator for systems with limited number of PMUs," IEEE Trans. Power Syst., vol. 30, pp. 1511-1517, Aug. 2015.

[18] M. Asprou, S. Chakrabarti, and E. Kyriakides, "A two-stage state estimator for dynamic monitoring of powersystems," IEEE Systems Journal, vol. PP, pp. 1-10, Dec. 2014.

[19] H. Karimipour and V. Dinavahi, "Extended kalman filter-based parallel dynamic state estimation," IEEE Trans. Smart Grid, vol. 6, pp. 1539-1549, Jan. 2015.

[20] J. Zhao, G. Zhang, K. Das, G. N. Korres, N. M. Manousakis, A. K.Sinha, and Z. He, "Power system real-time monitoring by using pmu-based robust state estimation method," IEEE Trans. Smart Grid, vol. pp, no. 99, pp. 1-10, May 2015.

[21] R. F. Nuqui, "State estimation and voltage security monitoring using synchronized phasor measurements," Ph.D. dissertation, Virginia Polytech. Inst. State Univ., Blacksburg, A., Jul 2001.

[22] M. Glavic and T. Van Cutsem, "Tracking network state from combined scada and synchronized phasor measurements," in Bulk Power Systems Dynamics and Control-IX (IREP), Rethymnon (Greece), Aug. 2013. [Online]. Available: http: //dx.doi.org/10.1109/IREP.2013.6629376

[23] B. A. Alcaide-Moreno, C. R. Fuerte-Esquivel, M. Glavic, and T. Van Cutsem, "Hybrid processing of scada and synchronized phasor measurements for tracking network state," in IEEE PES General Meeting, Denver (CO), Jul. 2015.

[24] K. D. Jones, A. Pal, and J. S. Thorp, "Methodology for performing synchrophasor data conditioning and validation," IEEE Trans. Power Syst., vol. 30, no. 3, pp. 1121-1130, May 2015.

[25] J. D. McDonald, Electric Power Substations Enginnering, Third Edition. CRC-Press, 2012.

[26] "Guideline for time synchronization of protection, control and monitoring," WECC Technical Operations Subcommittee, Tech. Rep., 2004. [Online]. Available: http:/www.wecc.biz/Reliability

[27] S. Makridakis and S. Wheelwright, Forecasting methods and applications. John Wiley and Sons, 1978.

[28] A. Gjelsvik, S. Aam, and L. Holten, "Hachtel's augmented matrix method: A rapid method improving numerical stability in power system static estimation," IEEE Trans. Power App. and Syst., vol. PAS-104, no. 11, pp. 2987-2993, Nov. 1985.

[29] "Test systems for voltage stability and security analysis - PES-TR19," IEEE PES, Tech. Rep., Aug. 2015. [Online]. Available: http://resourcecenter.ieee-pes.org/

[30] M. C. V. B. Naduvathparambil and A. Feliachi, "Communication delays in wide area measurement systems," in Proc. of 34th Southeastern Symposium on Systems Theory, Huntsville, USA, Mar. 2004, pp. 118-122.

[31] C. Ponce and D. Bindel, "Flier: Practical topology update detection using sparse pmus," IEEE Trans. Power Syst., In Press, 2017.

[32] C. Ponce, "Network-structured error flattening for power grids and other real-world networks," Ph.D. dissertation, Cornell University, Ithaca, N.Y., Jul May, 2016.

[33] S. Chakrabarti and E. Kyriakides, "Pmu measurement uncertainty considerations in wls state estimation," IEEE Trans. Power Syst., vol. 22, no. 2, pp. 1062-1071, May 2009. 